\title{
The VLA Nascent Disk And Multiplicity Survey of Perseus Protostars (VANDAM). III. Extended Radio Emission from Protostars in Perseus
}

\author{
Łukasz Tychoniec $^{1,2}$ (1D), John J. Tobin ${ }^{1,3}$ (10), Agata Karska ${ }^{4}$, Claire Chandler ${ }^{5}$ (D), Michael M. Dunham ${ }^{6,7}$ (1), Zhi-Yun Li ${ }^{8}$, \\ Leslie W. Looney ${ }^{9}$ (1), Dominique Segura-Cox ${ }^{9}$, Robert J. Harris ${ }^{9}$, Carl Melis ${ }^{10}{ }^{(1 D}$, and Sarah I. Sadavoy ${ }^{11}$ (1) \\ 1 Leiden Observatory, Leiden University, P.O. Box 9513, NL-2300RA, Leiden, The Netherlands \\ 2 Astronomical Observatory, Adam Mickiewicz University, Słoneczna 36, PL-60268 Poznań, Poland \\ ${ }^{3}$ Homer L. Dodge Department of Physics and Astronomy, University of Oklahoma, 440 W. Brooks Street, Norman, OK 73019, USA \\ ${ }^{4}$ Centre for Astronomy, Nicolaus Copernicus University in Toruń, Faculty of Physics, Astronomy and Informatics, Grudziądzka 5, PL-87100 Toruń, Poland \\ ${ }^{5}$ National Radio Astronomy Observatory, P.O. Box O, 1003 Lopezville Road, Socorro, NM 87801-0387, USA \\ ${ }^{6}$ Department of Physics, State University of New York Fredonia, Fredonia, NY 14063, USA \\ ${ }^{7}$ Harvard-Smithsonian Center for Astrophysics, 60 Garden Street, Cambridge, MA 02138, USA \\ ${ }^{8}$ Department of Astronomy, University of Virginia, Charlottesville, VA 22903, USA \\ ${ }^{9}$ Department of Astronomy, University of Illinois, Urbana, IL 61801, USA \\ ${ }^{10}$ Center for Astrophysics and Space Sciences, University of California, San Diego, CA 92093, USA \\ ${ }^{11}$ Max-Planck-Institut für Astronomie, Königstuhl 17, D-69117 Heidelberg, Germany \\ Received 2017 July 17; revised 2017 October 20; accepted 2017 November 7; published 2017 December 28
}

\begin{abstract}
Centimeter continuum emission from protostars offers insight into the innermost part of the outflows, as shock-ionized gas produces free-free emission. We observed a complete population of Class 0 and I protostars in the Perseus molecular cloud at 4.1 and $6.4 \mathrm{~cm}$ with resolution and sensitivity superior to previous surveys. From a total of 71 detections, eight sources exhibit resolved emission at $4.1 \mathrm{~cm}$ and $/$ or $6.4 \mathrm{~cm}$. In this paper, we focus on this subsample, analyzing their spectral indices along the jet and their alignment with respect to the large-scale molecular outflow. Spectral indices for fluxes integrated toward the position of the protostar are consistent with free-free thermal emission. The value of the spectral index along a radio jet decreases with distance from the protostar. For six sources, emission is well aligned with the outflow central axis, showing that we observe the ionized base of the jet. This is not the case for two sources, where we note misalignment of the emission with respect to the large-scale outflow. This might indicate that the emission does not originate in the radio jet, but rather in an ionized outflow cavity wall or disk surface. For five of the sources, the spectral indices along the jet decrease well below the thermal free-free limit of -0.1 with $>2 \sigma$ significance. This is indicative of synchrotron emission, meaning that high-energy electrons are being produced in the outflows close to the disk. This result can have far-reaching implications for the chemical composition of the embedded disks.
\end{abstract}

Key words: radio continuum: stars - stars: formation - stars: jets - stars: protostars - stars: winds, outflows techniques: interferometric

\section{Introduction}

Outflows and jets are ubiquitous phenomena in star formation. The youngest and most embedded Class 0 and Class I protostars are known to drive some of the most powerful outflows (Bontemps et al. 1996; Caratti o Garatti et al. 2012). Outflows can be observed across the electromagnetic spectrum as a HerbigHaro jet in the visible regime (e.g., Reipurth \& Cernicharo 1995; Bally et al. 1996); hot molecular and atomic gas in the near- and mid-infrared (e.g., Nisini et al. 2002); and cold entrained gas in the millimeter (e.g., Plunkett et al. 2013; Lee et al. 2015). At centimeter wavelengths, we are able to trace the free-free emission arising from the ionized gas in the outflows (Reynolds 1986; Anglada et al. 1998). Centimeter emission from protostars, if resolved, often appears extended, matching well with directions of the large-scale outflow (e.g., Rodríguez et al. 1986; Marti et al. 1993; Rodríguez 1994; Anglada 1995). Thus, it is inferred that radio jets trace regions that constitute the base of the outflow, and the correlation of radio emission with outflow force provides strong evidence for this link (Cabrit \& Bertout 1992; Anglada 1995; Wu et al. 2004; Shirley et al. 2007)

With the spectral index at centimeter wavelengths, we can discriminate between mechanisms responsible for the observed emission. Thermal free-free emission is expected to have flat or positive spectral index $(-0.1 \leqslant \alpha<2.0$; Panagia \& Felli 1975; Rodríguez et al. 1993) varying with the optical depth. Similar or steeper indices are expected for thermal dust emission, but it does not contribute significantly to the radio emission above $4 \mathrm{~cm}$ because for typical dust masses and opacities in the lowmass, star-forming regions, especially for the youngest protostars, the emission is $\sim 10 \times$ below the sensitivity limit (Tobin et al. 2015). That emission becomes significant as the protostellar disk evolves and the grain growth proceeds (e.g., Wilner et al. 2005). Negative spectral indices $(\alpha<-0.1)$ are a manifestation of a non-thermal emission.

Positive spectral indices are most frequently observed from young Class 0/I protostars, while for more evolved Class II/III young stellar objects, coronal activity commonly produces nonthermal radiation (e.g., Dzib et al. 2013; Pech et al. 2016). However, there are a growing number of sources with observed negative spectral indices in the outflow positions of young protostars. Since first observed in the protostellar outflows, negative spectral indices were explained by synchrotron emission, with shocks as a mechanism to accelerate electrons (Rodríguez et al. 1989). Curiel et al. (1993) observed significant variations of the spectral index along the jet of the Serpens SMM1 protostar, also known as a Serpens triple radio source (e.g., Snell \& Bally 1986), and suggested that both free-free and synchrotron emission mechanisms can simultaneously operate in outflows. Direct confirmation of synchrotron emission contributing to at least a part of the radio flux from protostars was provided by detecting 
linear polarization in the $\mathrm{HH}$ 80-81 outflows of a massive protostar IRAS 18162-2048 (Carrasco-González et al. 2010). Ainsworth et al. (2014) also observed synchrotron emission at the bow shock position in the jet from DG Tau, a solar-mass, pre-main sequence star, suggesting that synchrotron emission phenomenon can be observed even in the outflows of low-mass stars.

Resolved radio jets were observed in many protostars; however, the sample of the low-mass sources with luminosities $\sim 1 L_{\odot}$ is still sparse. One of the rare cases is SVS $13 \mathrm{C}$ (Rodríguez et al. 1997), with bolometric luminosity of $1.5 L_{\odot}$, which we also present in this paper. Centimeter radio emission is correlated with the bolometric luminosity (Cabrit \& Bertout 1992; Anglada 1995; Shirley et al. 2007); thus, $\sim 1 L_{\odot}$ sources require higher sensitivity than massive protostars to have their radio emission detected. Also, if we want to observe the region closest to the protostar, we must resolve where the jet is being collimated, so resolution well below arcsecond is necessary. Most previous radio observations found extended radio jets only toward higher luminosity sources, and there have only been a few surveys with high sensitivity, but not subarcsecond resolution, or probing a selected sample of protostars in different regions, instead of surveying complete population in one cloud. Rodríguez et al. (1999) achieved sensitivity up to $10 \mu \mathrm{Jy}$, with observations at 3.6 and $6 \mathrm{~cm}$, but their sample was limited to the central part of the NGC 1333 region in Perseus and the resolution was around 5". The AMI Consortium: Scaife et al. (2011a, 2011b, 2012a, 2012b) observed selected protostars in Perseus, Serpens, Taurus, and several isolated cores at low resolution $\left(30^{\prime \prime}\right)$ and sensitivity up to $15 \mu \mathrm{Jy}$ at $1.8 \mathrm{~cm}$. The Gould's Belt Very Large Array (VLA) Survey targeted large sample of protostars in selected star-forming regions with $16 \mu \mathrm{Jy}$ sensitivity and resolution up to 0". 4 (Dzib et al. 2013, 2015; Kounkel et al. 2014; Ortiz-León et al. 2015; Pech et al. 2016) in C-band (4.1 and $6.4 \mathrm{~cm})$. Reipurth et al. (2002, 2004) surveyed a sample of protostars in different star-forming regions with high resolution $(0 . " 35)$ and sensitivity $(\sim 10 \mu \mathrm{Jy})$ at $3.6 \mathrm{~cm}$ and found evidence for a few modestly extended jets.

To further investigate the properties of the protostellar jets and to overcome limitations of the previous surveys, we use VLA Nascent Disk and Multiplicity (VANDAM) survey C-band observations of all known (84) Class 0/I protostars in the Perseus molecular cloud, one of the most active stellar nurseries in the solar neighborhood $(d \sim 230 \mathrm{pc}$, Hirota et al. 2008). VANDAM is the largest radio survey of the youngest (Class 0 and I) protostars in a single cloud ever undertaken, with high resolution up to $\sim 80$ au $(0$ !" 3$)$ at the distance to Perseus and remarkable sensitivity of $\sim 5 \mu \mathrm{Jy}$ in the C-band, both superior to previous surveys. Protostars targeted by the survey span the low-mass regime with luminosities between $0.1 L_{\odot}$ and $30 L_{\odot}$. A comprehensive study of the C-band observations will be the scope of a future paper (Ł. Tychoniec et al. 2018, in preparation). Here, we present a subset of sources with resolved radio jets. We will investigate their properties and spectral index distributions along the jet and discuss their relation to the larger-scale outflows.

\section{Observations}

Observations were a part of the VANDAM Survey. ${ }^{12}$ Observations were taken in A configuration between 2014

\footnotetext{
${ }^{12}$ FITS files from both $\mathrm{Ka}$ and $\mathrm{C}$-band observations will be available at https://dataverse.harvard.edu/dataverse/VANDAM.
}

February 28 and April 12, with 3C48 as an absolute flux and bandpass calibrator and $\mathrm{J} 0336+3218$ as the complex gain calibrator. The observations were performed in 8-bit mode, resulting in $2 \mathrm{GHz}$ of bandwidth divided into $16128 \mathrm{MHz}$ subbands with $2 \mathrm{MHz}$ channels and full polarization products recorded; the polarization results will be published in a future paper. The $2 \mathrm{GHz}$ is divided into two $1 \mathrm{GHz}$ basebands that were centered at $4.7 \mathrm{GHz}(6.4 \mathrm{~cm})$ and $7.3 \mathrm{GHz}(4.1 \mathrm{~cm})$. The basebands were centered away from the band edges to avoid persistent radio frequency interference. The absolute flux uncertainty is estimated to be $\sim 5 \%$. With the simultaneous observations at the two ends of C-band, the limiting factor in our ability to determine the spectral index is the accuracy of the flux density model of 3C48 which is known to better than $\sim 2 \%$ (Perley \& Butler 2017).

The data were reduced and calibrated using CASA 4.1.0 (McMullin et al. 2007) and version 1.2.2 of the VLA Pipeline. Further flagging was conducted after the pipeline run and is detailed further in Tobin et al. (2015). We imaged the data using the clean task in CASA version 4.1.0 with both natural weighting and Briggs weighting with the robust parameters set to 0.25 . In all cases, we imaged the full field of the data set using images with dimensions either $8192^{2}$ or $16384^{2}$ pixels and pixel widths of $0 . " 05$. The nominal angular resolution at $4.1 \mathrm{~cm}$ was $\sim 0$ ". 30 and 0 ". 25 for natural and robust $=0.25$ weighting, respectively; the angular resolution at $6.4 \mathrm{~cm}$ was $\sim 0$." 50 and 0."35. For some images with bright extragalactic sources, we performed two iterations of phase-only selfcalibration to achieve better dynamic range. Self-calibration was not necessary for any fields containing resolved jets.

\section{Results}

From the 71 protostars detected in the C-band, we identified eight that had clearly extended emission as listed in Table 1 . The integrated flux densities were obtained by fitting the 2D Gaussian function to the sources with CASA imfit task. In most cases, a multiple Gaussian fit was necessary to obtain as small a residual as possible. The integrated flux value is then the sum of all Gaussians. Uncertainty of the integrated flux was obtained from imfit task. Peak flux density and the rms were measured with CASA imstat task. Both values and their uncertainties were extracted from the images before primary beam correction and subsequently corrected for primary beam response measured at the position of the source. For each source, we calculated spectral index values of both integrated and peak flux using the following equation:

$$
\alpha_{4.1 / 6.4}=\frac{\log \left(F_{4.1} / F_{6.4}\right)}{\log (6.4 / 4.1)},
$$

where $F_{4.1}$ and $F_{6.4}$ are integrated or peak fluxes at $4.1 \mathrm{~cm}$ and $6.4 \mathrm{~cm}$, respectively. Uncertainty of the spectral index was obtained using standard error propagation (Chiang et al. 2012). Our analysis was done primarily on robust $=0.25$ images, except for Per-emb-33 and SVS 13C, which have the most extended radio emission and hence the natural weighting was more appropriate. A summary of the measured properties of the protostars with resolved jets is presented in Table 2 .

We measured spectral indices at both central and off-source positions along the resolved jets. This was done by taking a median of 4.1 and $6.4 \mathrm{~cm}$ flux from area of the size of synthesized beam using the CASA imstat task and then calculating the spectral index in the way described above with the rms of the image used as a flux uncertainty. Positions where the spectral index was measured are 
Table 1

Properties of Powering Sources of Resolved Jets

\begin{tabular}{|c|c|c|c|c|c|c|}
\hline Source & Region & Other Names $^{\mathrm{a}}$ & Class $^{\mathrm{b}}$ & $\begin{array}{l}L_{\mathrm{bol}}^{b} \\
\left(L_{\odot}\right)\end{array}$ & $\begin{array}{l}T_{\mathrm{bol}}^{b} \\
(\mathrm{~K})\end{array}$ & $\begin{array}{c}\mathrm{PA}^{\mathrm{c}} \\
\left({ }^{\circ}\right)\end{array}$ \\
\hline Per-emb-8 & IC 348 & PER22, IC 348a, IRAS $03415+3152$, YSO 48 & 0 & $2.6 \pm 0.5$ & $43.0 \pm 6.0$ & $135(5)$ \\
\hline Per-emb-18 & NGC 1333 & NGC 1333 IRAS7, YSO 24 & 0 & $2.8 \pm 1.7$ & $59.0 \pm 12.0$ & $159(2)$ \\
\hline Per-emb-20 & L1455 & L1455 IRS4 & 0 & $1.4 \pm 0.2$ & $65.0 \pm 3.0$ & $115(2)$ \\
\hline Per-emb-30 & B1 & PER19, B1 SMM11, YSO 40 & $0 / \mathrm{I}$ & $1.1 \pm 0.0$ & $93.0 \pm 6.0$ & $109(2)$ \\
\hline Per-emb-33 & L1448 & PER02, L1448 N(A), L1448 IRS 3B, YSO 2 & 0 & $8.3 \pm 0.8$ & $57.0 \pm 3.0$ & $105(3)$ \\
\hline Per-emb-36 & NGC 1333 & PER06, NGC 1333 IRAS 2B, YSO 16 & I & $6.9 \pm 1.0$ & $85.0 \pm 12.0$ & $24(1)$ \\
\hline L1448 IRS 3A & L1448 & $\cdots$ & I & $9.2 \pm 1.3$ & $47.0 \pm 2.0$ & $38(4)$ \\
\hline SVS 13C & NGC 1333 & VLA2 & 0 & $1.5 \pm 0.2$ & $21.0 \pm 1.0$ & $8(1)$ \\
\hline
\end{tabular}

Notes.

a Names: YSOXX (Jørgensen et al. 2007), PERXX (Karska et al. 2014), VLAXX (Rodríguez et al. 1997).

b References: Enoch et al. 2009; Sadavoy et al. 2014.

${ }^{\mathrm{c}}$ References: (1) Plunkett et al. 2013; (2) Davis et al. 2008; (3) Kwon et al. 2006; (4) Lee et al. 2016; (5) J. Tobin et al. 2018, in preparation.

showed in the Appendix. Using the area of the synthesized beam takes into account the resolution of the observations, but as the emission fades steeply with distance to the protostar, we use median as less sensitive to those outliers. While some of the measured beams are overlapping, making measurements not entirely independent, we note that the trends seen in the spectral index maps are consistent with the obtained values. Protostellar positions are adopted from the Ka-band survey results that identify the protostar positions with the highest accuracy (Tobin et al. 2016b). To measure the direction of the radio jet, we obtain the position angle of a single 2D Gaussian fit to the source and summarize the results in Table 3. For all sources, we produced a spectral index map. To obtain the same angular resolution at both wavelengths, the $4.1 \mathrm{~cm}$ frames were convolved with the same restoring beam as $6.4 \mathrm{~cm}$ frames in the clean procedure in CASA. Pixels with values below $3 \times$ rms were masked.

We do not expect the dust emission to significantly contribute to the C-band flux. The values of the spectral indices at Ka-band for all sources presented here are found below the value expected from the thermal dust emission (Tobin et al. 2016b), which shows that free-free emission is dominating the C-band flux and also significantly affects the Ka-band fluxes. We also note that any contribution of the thermal dust emission would be limited the the central source position. Regarding the detected negative spectral indices, any thermal dust contribution would increase the value of spectral index between 4.1 and $6.4 \mathrm{~cm}$, thus any potential contribution does not cast a doubt on the tentative detection of the synchrotron emission.

We detected eight protostars with resolved radio jets extended on scales between 80 and 900 au. For all of the objects, the spectral indices at the position of the protostar are consistent with free-free emission. For five sources, we detect negative spectral indices along the radio jet/outflow direction, possibly indicative of synchrotron emission. We also find that the integrated spectral indices of extended jet sources are lower than the median observed for the whole sample $\left(\alpha_{\text {med }}=0.51\right.$; Ł. Tychoniec et al. 2018, in preparation). The significant contribution of radio flux from the more optically thin extended jets in the form of free-free or synchrotron emission would lower the value of the overall spectral index.

Extended emission is observed from protostars with bolometric luminosities between $1 L_{\odot}$ and $9.2 L_{\odot}$. The sample includes both Class 0 and Class I protostars with low bolometric temperatures of $T_{\text {bol }}<100 \mathrm{~K}$, meaning that the protostars are all relatively young (e.g., Chen et al. 1995; Enoch et al. 2009).
Other than this upper limit on $T_{\text {bol }}$, there are no specific trends between protostar properties and extended radio emission. We will discuss the results for each source individually.

\subsection{Per-emb-36}

Per-emb-36 (also known as NGC 1333 IRAS 2B; Figure 1) is a Class I system comprised of two protostars separated by $\sim 70$ au (Tobin et al. 2016b). Centimeter emission is dominated by the bipolar jet from Per-emb-36-A. Per-emb-36-B is not clearly resolved from Per-emb-36-A at $4.1 \mathrm{~cm}$ or $6.4 \mathrm{~cm}$, but a small asymmetry in the $4.1 \mathrm{~cm}$ emission is observed toward the position of Per-emb-36-B as identified at Ka-band. There is a clear decrease in a spectral index along the outflow direction, from $0.67 \pm 0.18$ at the protostellar position to $-0.64 \pm 0.20$ in the northern outflow. The position angle of the extended emission is $24^{\circ} \pm 2^{\circ}$, closely matching the position angle of the $\mathrm{CO}$ outflow observed by Plunkett et al. (2013). This is a strong indication that the jet from the protostar is producing the extended radio emission. The outflow is asymmetric, showing stronger emission from the northern part, and it is only on this side that we detect a steep spectral index that likely indicates the presence of the synchrotron emission. It is noteworthy that the $6.4 \mathrm{~cm}$ emission peaks significantly away from the source at 0." 4 (93 au), which is not the case for any other sources we observed. Shift is also clearly seen in full-bandwidth image (Figure 9 in the Appendix). We estimate the error of the position measurement at $6.4 \mathrm{~cm}$, following Equation (1) in Condon et al. (1998), and the resulting error is below $0 !^{\prime \prime} 01$ in each coordinate, showing that this displacement is robustly detected. It is possible that this offset in the peak emission at $6.4 \mathrm{~cm}$ is due to a strong bow shock producing synchrotron emission, which may have a counterpart in X-rays similar to DG Tau (Ainsworth et al. 2014). We examined the literature for possible X-ray detections and find an X-ray source (Preibisch 1997) near Per-emb-36. However, the X-ray emission may be from a Class III (BD +30 547) source with a projected separation of $\sim 4^{\prime \prime}$ from Per-emb-36; the source(s) were detected toward the edge of the X-ray observations field where the positional uncertainty is large.

\subsection{SVS $13 C$}

SVS 13C (Figure 2) is a Class 0 object, as classified by Sadavoy et al. (2014), located in the NGC 1333 region in Perseus. This source was also detected at millimeter wavelengths by Looney et al. (2000). It produces the most prominent extended 
Table 2

C-band Observation Results

\begin{tabular}{|c|c|c|c|c|c|c|c|c|c|c|}
\hline Source & $\begin{array}{l}\text { R.A. } \\
\text { (J2000) }\end{array}$ & $\begin{array}{l}\text { Decl. } \\
\text { (J2000) }\end{array}$ & $\begin{array}{c}F_{\nu, \text { int }} \\
(6.4 \mathrm{~cm}) \\
(\mathrm{mJy})\end{array}$ & $\begin{array}{c}F_{\nu, \text { peak }} \\
(6.4 \mathrm{~cm}) \\
\left(\mathrm{mJy} \mathrm{bm}^{-1}\right)\end{array}$ & $\begin{array}{c}\mathrm{rms} \\
(6.4 \mathrm{~cm}) \\
\left(\mathrm{mJy} \mathrm{bm}^{-1}\right)\end{array}$ & $\begin{array}{c}F_{\nu, \text { int }} \\
(4 \mathrm{~cm}) \\
(\mathrm{mJy})\end{array}$ & $\begin{array}{c}F_{\nu, \text { peak }} \\
(4 \mathrm{~cm}) \\
\left(\mathrm{mJy} \mathrm{bm}^{-1}\right)\end{array}$ & $\begin{array}{c}\mathrm{rms} \\
(4 \mathrm{~cm}) \\
\left(\mathrm{mJy} \mathrm{bm}^{-1}\right)\end{array}$ & $\begin{array}{l}\text { Sp. Index } \\
\text { Int. }\end{array}$ & $\begin{array}{l}\text { Sp. Index } \\
\text { Peak }\end{array}$ \\
\hline Per-emb-8 & 03:44:43.981 & $+32: 01: 35.210$ & $0.2788 \pm 0.0122$ & 0.1323 & 0.0049 & $0.3132 \pm 0.0166$ & 0.1240 & 0.0037 & $0.26 \pm 0.15$ & $-0.14 \pm 0.10$ \\
\hline Per-emb-18 & 03:29:11.258 & $+31: 18: 31.072$ & $0.1919 \pm 0.0090$ & 0.1326 & 0.0058 & $0.1957 \pm 0.0059$ & 0.1505 & 0.0043 & $0.04 \pm 0.12$ & $0.28 \pm 0.11$ \\
\hline Per-emb-20 & $03: 27: 43.276$ & $+30: 12: 28.780$ & $0.1477 \pm 0.0083$ & 0.1093 & 0.0054 & $0.1384 \pm 0.0124$ & 0.1098 & 0.0042 & $-0.14 \pm 0.23$ & $0.01 \pm 0.14$ \\
\hline Per-emb-30 & 03:33:27.303 & $+31: 07: 10.159$ & $0.2747 \pm 0.0171$ & 0.1542 & 0.0055 & $0.2815 \pm 0.0254$ & 0.1681 & 0.0055 & $0.05 \pm 0.24$ & $0.19 \pm 0.11$ \\
\hline Per-emb-33 & 03:25:36.379 & $+30: 45: 14.727$ & $0.1648 \pm 0.0139$ & 0.1154 & 0.0053 & $0.1386 \pm 0.0071$ & 0.1041 & 0.0042 & $-0.38 \pm 0.22$ & $-0.23 \pm 0.13$ \\
\hline Per-emb-33-A & $03: 25: 36.312$ & $+30: 45: 15.153$ & $0.1389 \pm 0.0082$ & 0.1154 & 0.0053 & $0.1386 \pm 0.0071$ & 0.1041 & 0.0042 & $-0.00 \pm 0.17$ & $-0.23 \pm 0.13$ \\
\hline Per-emb-33-B & $03: 25: 36.321$ & $+30: 45: 14.913$ & $<0.0158 \pm 0.0053$ & $<0.0158$ & 0.0053 & $<0.0127 \pm 0.0042$ & $<0.0127$ & 0.0042 & $-99.00 \pm-99.00$ & $-99.00 \pm-99.00$ \\
\hline Per-emb-33-C & $03: 25: 36.380$ & $+30: 45: 14.722$ & $0.0259 \pm 0.0057$ & 0.0282 & 0.0053 & $<0.0127 \pm 0.0042$ & $<0.0127$ & 0.0042 & $<-1.57 \pm 0.88$ & $<-1.75 \pm 0.84$ \\
\hline Per-emb-36-A & $03: 28: 57.373$ & $+31: 14: 15.764$ & $0.2433 \pm 0.0192$ & 0.1267 & 0.0051 & $0.2523 \pm 0.0169$ & 0.1037 & 0.0044 & $0.08 \pm 0.23$ & $-0.44 \pm 0.13$ \\
\hline Per-emb-36-B & 03:28:57.370 & $+31: 14: 16.072$ & $<0.0152 \pm 0.0051$ & $<0.0152$ & 0.0051 & $<0.0132 \pm 0.0044$ & $<0.0132$ & 0.0044 & $-99.00 \pm-99.00$ & $-99.00 \pm-99.00$ \\
\hline L1448 IRS3A & 03:25:36.499 & $+30: 45: 21.880$ & $0.4922 \pm 0.0149$ & 0.3717 & 0.0053 & $0.5074 \pm 0.0149$ & 0.3607 & 0.0043 & $0.07 \pm 0.09$ & $-0.07 \pm 0.04$ \\
\hline SVS $13 \mathrm{C}$ & 03:29:01.970 & $+31: 15: 38.053$ & $1.0676 \pm 0.0218$ & 0.6812 & 0.0049 & $1.2095 \pm 0.0296$ & 0.6753 & 0.0039 & $0.28 \pm 0.07$ & $-0.02 \pm 0.02$ \\
\hline
\end{tabular}

Notes.

Integrated spectral index: calculated with flux density integrated over the full extent of the source.

${ }^{\mathrm{b}}$ Peak spectral index: calculated with peak value of the flux density. 
Table 3

Spectral Indices at Different Positions and Position Angles of Resolved Emission

\begin{tabular}{|c|c|c|c|c|c|}
\hline Source & $\begin{array}{l}\mathrm{PA}^{\mathrm{a}} \\
\mathrm{deg}\end{array}$ & $\begin{array}{c}\text { Sp. Index } \\
\text { Central }\end{array}$ & $\begin{array}{l}\text { Sp. Index } \\
\text { Outflow } 1\end{array}$ & $\begin{array}{l}\text { Sp. Index } \\
\text { Outflow } 2\end{array}$ & $\begin{array}{l}\text { Sp. Index } \\
\text { Outflow } 3\end{array}$ \\
\hline Per-emb-8 & $13.0 \pm 1.9$ & $0.16 \pm 0.12$ & $-0.25 \pm 0.16$ & $\ldots$ & $\cdots$ \\
\hline Per-emb-18 & $163.9 \pm 15.6$ & $0.37 \pm 0.14$ & $-0.76 \pm 0.94$ & $-1.13 \pm 0.42$ & $\cdots$ \\
\hline Per-emb-30 & $123.8 \pm 2.9$ & $0.28 \pm 0.14$ & $-0.20 \pm 0.46$ & $-1.10 \pm 0.47$ & $\cdots$ \\
\hline Per-emb-33 & $108.0 \pm 3.7$ & $-0.14 \pm 0.14$ & $-0.30 \pm 0.74$ & $-0.27 \pm 0.52$ & $-0.53 \pm 0.50$ \\
\hline Per-emb-36 & $20.6 \pm 1.7$ & $0.67 \pm 0.18$ & $-0.64 \pm 0.20$ & $-0.20 \pm 0.28$ & $\cdots$ \\
\hline
\end{tabular}

Note. Outflow positions where spectral index was measured are numbered with descending declination. See figures in the Appendix.

${ }^{\text {a }}$ Position angle measured from north to east.
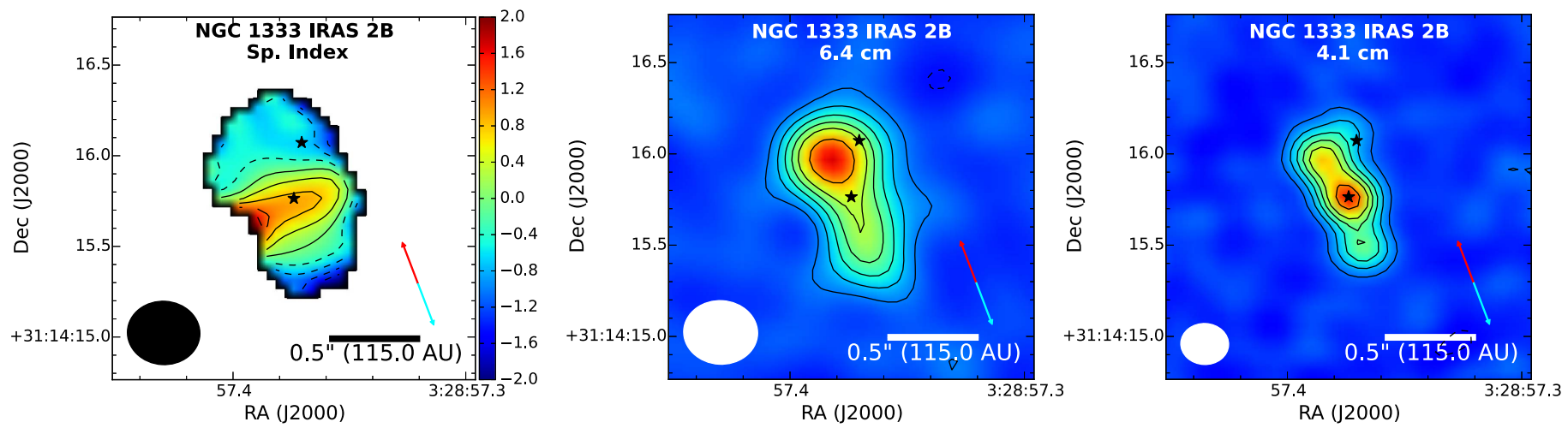

Figure 1. Images of Per-emb-36. Spectral index map with contours: $[-2,-1.6,-1.2,-0.8,-0.4,0.0,0.4,0.8,1.2,1.6,2.0] .6 .4$ and $4.1 \mathrm{~cm}$ maps with contours $[-3$, $3,6,9,12,15,20] \times \sigma$ where $\sigma_{6.4 \mathrm{~cm}}=4.83 \mu \mathrm{Jy}$ and $\sigma_{4.1 \mathrm{~cm}}=3.90 \mu \mathrm{Jy}$. Synthesized beam is shown in the left bottom corner (Sp. Index and $6.4 \mathrm{~cm}: 0$.! $41 \times 0$ ". 35 , $4.1 \mathrm{~cm}: 0$ !' $26 \times 0$ !' 22 ). The stars mark the position of the protostars based on Ka-band observations (Tobin et al. 2016b) and the red and blue arrows indicate outflow direction from Plunkett et al. (2013) with a position angle $24^{\circ} .6 .4$ and $4.1 \mathrm{~cm}$ maps are not corrected for a primary beam response (spectral index derived from PB corrected map).
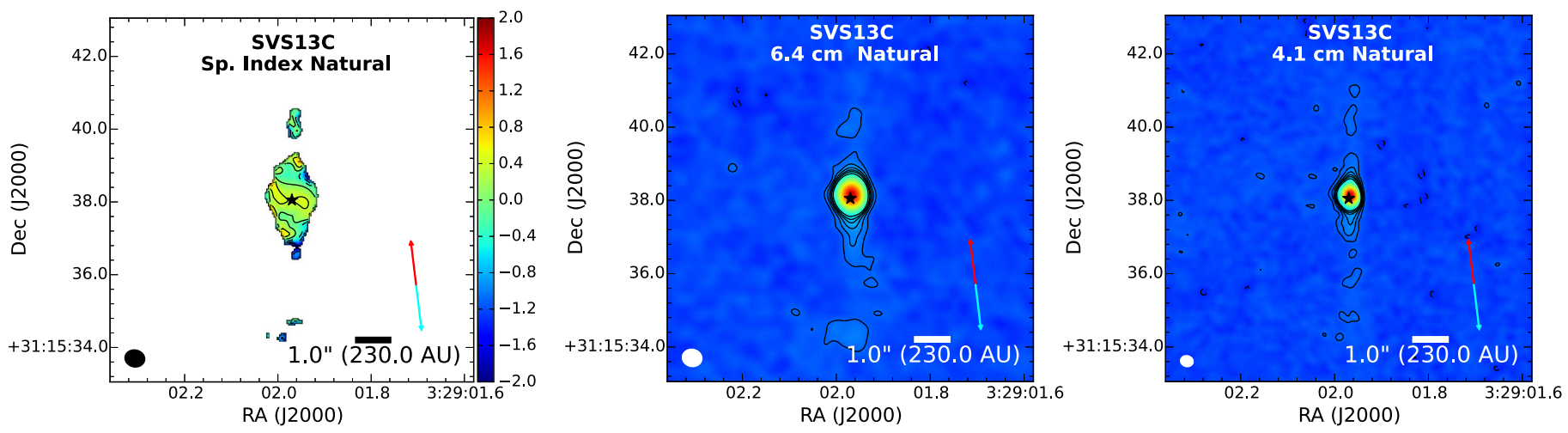

Figure 2. Naturally weighted map of SVS $13 \mathrm{C}$ with contours as in Figure $1\left(\sigma_{6.4 \mathrm{~cm}}=4.83 \mu \mathrm{Jy}\right.$ and $\left.\sigma_{4.1 \mathrm{~cm}}=3.90 \mu \mathrm{Jy}\right)$. Synthesized beam is shown in the left bottom corner (Sp. Index and $6.4 \mathrm{~cm}: 0 . ! 41 \times 0 . .35,4.1 \mathrm{~cm}: 0 ! .26 \times 0 ! .22$ ). The star marks the position of the protostar based on Ka-band observations (Tobin et al. $2016 \mathrm{~b}$ ) and the red and blue arrows indicate outflow direction from Plunkett et al. (2013) and Lee et al. (2016).

radio jet in our sample. The position angle for its centimeter emission is $9^{\circ} \pm 1^{\circ}$. It extends $\sim 4^{\prime \prime}(900$ au) in both the north and south directions. The extended centimeter emission toward this source had been previously reported by Rodríguez et al. (1997) (their source VLA2), and later by Reipurth et al. (2002) and Carrasco-González et al. (2008). However, our observations are the highest fidelity maps to date taken toward this source. We find an irregular, clumpy structure along the jet, with the spectral index significantly decreasing outward. At $2^{\prime \prime}$ (460 au) distance in the northern direction, we can observe a source of emission detached from the main component, suggesting that there is a clump of denser matter in the area. Plunkett et al. (2013) tentatively identified an outflow with $8^{\circ}$ position angle, and Lee et al. (2016) found a similar position angle of $0^{\circ}$, both of which are consistent with an extended radio jet. The outflow also appeared to be in the plane of the sky in the observations from both Plunkett et al. (2013) and Lee et al. (2016). This is consistent with the large proper motions of $\sim 100 \mathrm{~km} \mathrm{~s}^{-1}$ found by Raga et al. (2013).

\subsection{Per-emb-30}

Per-emb-30 (Figure 3) is a Class 0 source located in the Barnard 1 region. We find radio emission at $6.4 \mathrm{~cm}$ extending $\sim 1^{\prime \prime}$ 

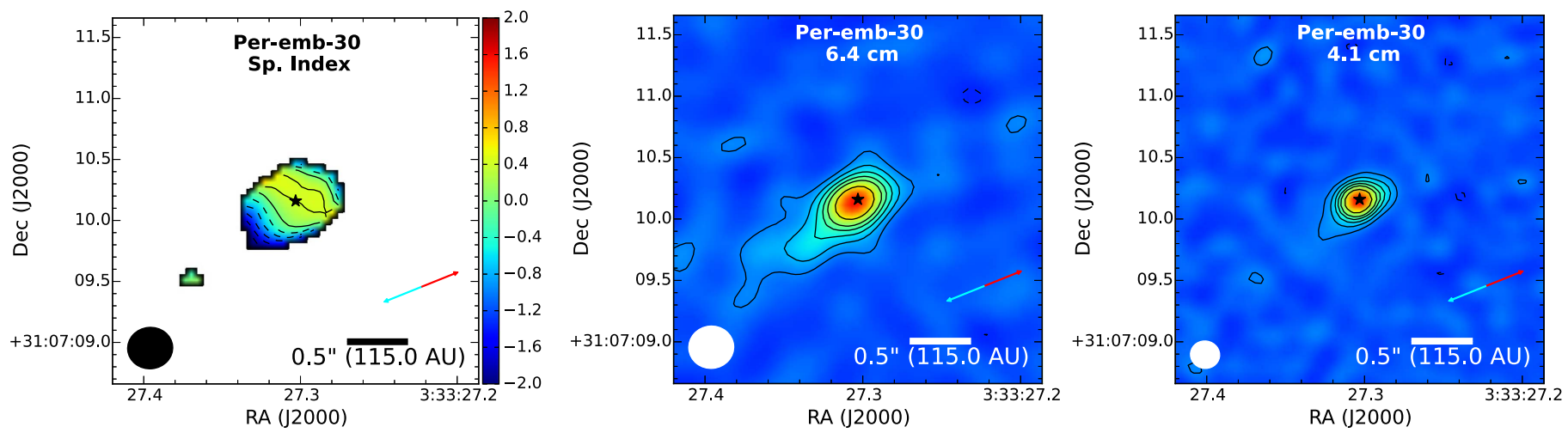

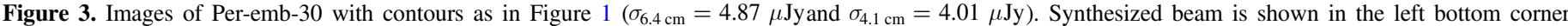

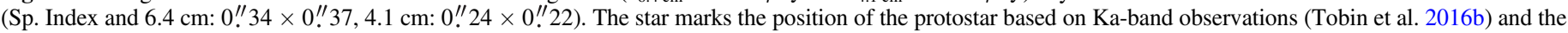
red and blue arrows indicate the outflow direction from Davis et al. (2008) with a position angle $109^{\circ}$.
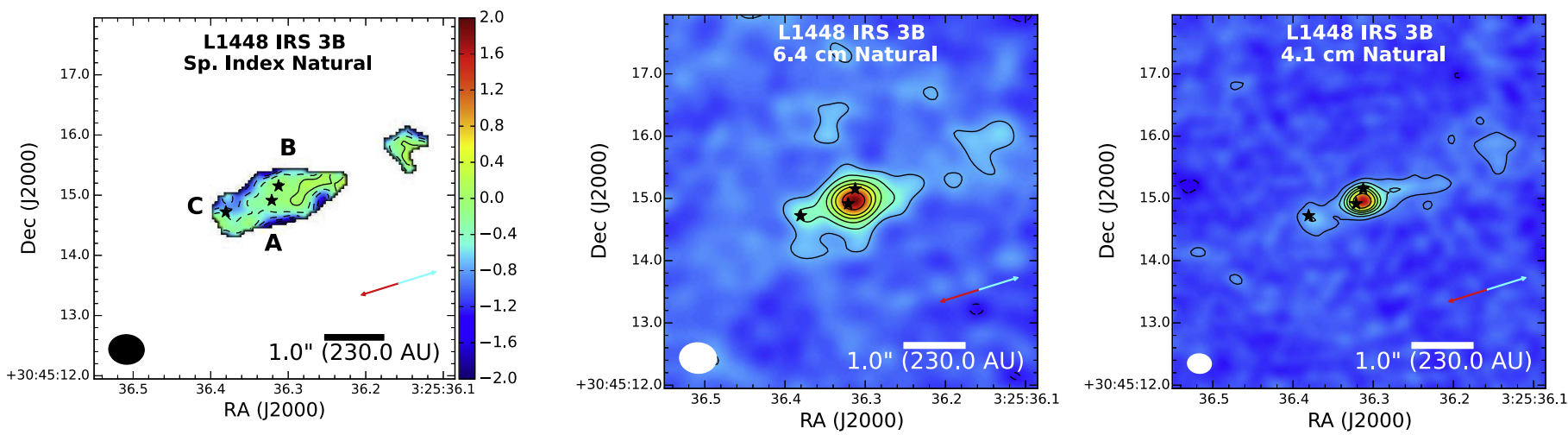

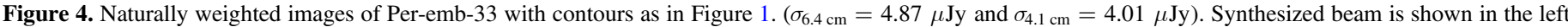

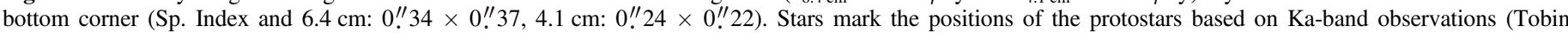

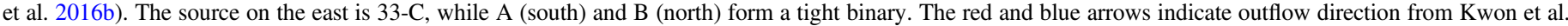
(2006) with a position angle of $105^{\circ}$.

(230 au) from the source and more compact emission at $4.1 \mathrm{~cm}$. The more compact emission at $4.1 \mathrm{~cm}$ results in a strongly negative spectral index $(-1.10 \pm 0.47)$ along the jet direction. The direction of extended radio emission is consistent with the $\mathrm{H}_{2}$ feature (HH790) originating from this source (Davis et al. 2008), having a position angle of $\sim 109^{\circ}$, which is consistent with our fit of $117^{\circ} \pm 4^{\circ}$. Storm et al. (2014) also finds evidence for an $\mathrm{HCO}^{+}$outflow toward Per-emb-30 with a similar position angle. Interestingly, the $\mathrm{H}_{2}$, radio, and $\mathrm{HCO}^{+}$outflow appear monopolar; upcoming $\mathrm{CO}$ observations from the MASSES survey (e.g., Lee et al. 2014) will be more definitive. The monopolar appearance may reflect the spatial distribution of dense gas around the protostar; the southeastern portion of the outflow is interacting with ambient medium, while the northwestern portion is not.

\subsection{Per-emb-33}

Per-emb-33 (Figure 4) is a triple system of Class 0 protostars, also known as L1448 IRS 3B (Looney et al. 2000) and L1448 N(B) (Curiel et al. 1990). Per-emb-33-A is dominating the emission in the C-band and Per-emb-33-C is marginally detected, in contrast to the $9 \mathrm{~mm}$ observations, where source $\mathrm{C}$ has the highest flux density (Tobin et al. 2016b). The resolution was not sufficient to resolve Per-emb-33-B from source A. The radio emission is also extended along the jet outflow direction measured by Lee et al. (2015), making it difficult to determine whether Peremb-33-C is actually detected or if this is just the extended jet emission. Along the radio jet/outflow direction to the west, there is a clump of emission separated from the source position by a distance 2". 4 (540 au), which also appears to be due to the radio jet. The spectral index along the outflow is consistent with optically thin free-free emission, varying from $-0.14 \pm 0.14$ in the A source position to $-0.30 \pm 0.74$ in the northwestern clump and $-0.53 \pm 0.50$ in the position of source C. Position angle of the extended jet $107^{\circ} \pm 5^{\circ}$ is consistent with the $\mathrm{CO}$ outflow position angle obtained by Kwon et al. (2006) and (Lee et al. 2015). Tobin et al. (2016a) find that the rotational center of the system is located closest to Per-emb-33-A, consistent with it driving the radio jet. They also showed that Per-emb-33-C drives a distinct, collimated outflow.

\section{5. $L 1448 \operatorname{IRS} 3 A$}

L1448 IRS 3A (Figure 5) is a Class I protostar and a wide companion of Per-emb-33, separated by 7 !! 3 ( 1700 au; Looney et al. 2000; Tobin et al. 2016b). It has extended centimeter emission in the western direction at both 4.1 and $6.4 \mathrm{~cm}$. The spectral index northeast of the protostar position is $-0.61 \pm 0.24$. This indicates that the emission could be produced by synchrotron emission, but the fact that emission arises on the edge of the source casts a doubt on this detection. The portion more extended to the west has a spectral index of $-0.13 \pm 0.12$ and is consistent with free-free emission. Measured position angle $\left(79^{\circ} \pm 1^{\circ}\right)$ of the 4.1 and $6.4 \mathrm{~cm}$ emission is notably different from the $\mathrm{CO}$ outflow position angle of $38^{\circ}$ measured by Lee et al. (2015). Recent ALMA observations show a disk in dust emission aligned 

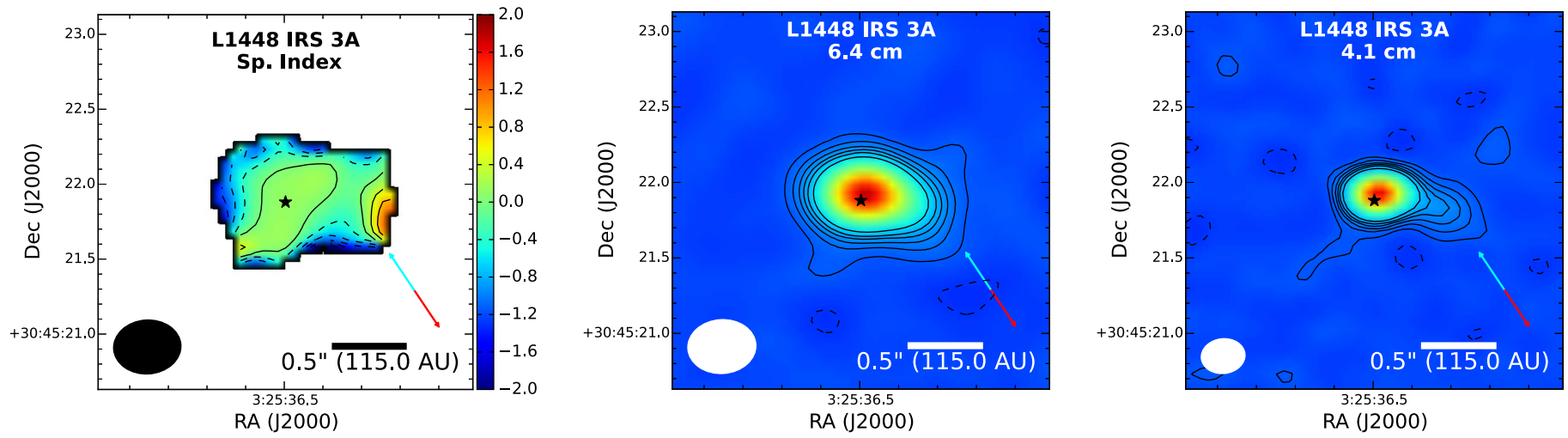

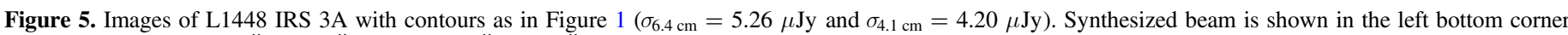

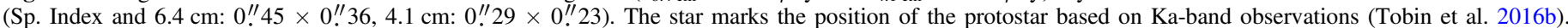
Red and blue arrows indicate outflow direction from Lee et al. (2015) with a position angle of $38^{\circ}$.
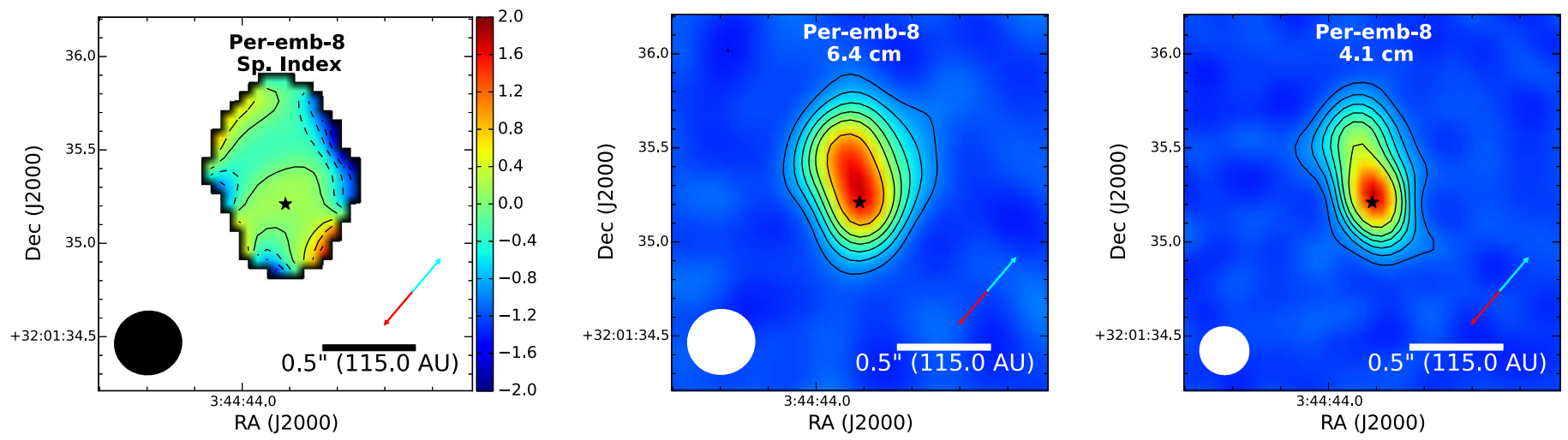

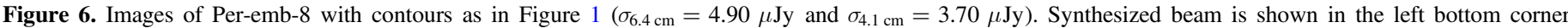

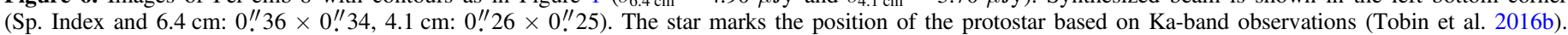
Red and blue arrows are orthogonal to the major axis of the disk based on ALMA observations (J. Tobin et al. 2018, in preparation).

perpendicularly to the CO outflow (J. Tobin et al. 2018, in preparation); thus, the difference in PA between the centimeter radio and the molecular outflow could indicate that the free-free emission is tracing a portion of an ionized outflow cavity. This may be similar to what was observed toward a more luminous protostar in Serpens (Hull et al. 2016). The $9 \mathrm{~mm}$ observations of L1448 IRS 3A are also extended in the same direction and the spectral index was found to be relatively flat (Tobin et al. 2016b). Thus, the free-free emission is likely contributing also at shorter wavelengths.

\subsection{Per-emb-8}

Per-emb-8 (Figure 6) is a Class 0 protostar in the IC 348 region and is located 9".6 ( 2200 au) from Per-emb-55, which is a close multiple itself (Tobin et al. 2016b). Per-emb-8 shows 4.1 and $6.4 \mathrm{~cm}$ emission extended north and slightly east, the position angle is $13^{\circ} \pm 2^{\circ}$. The spectral index smoothly decreases further away from the protostar, with a value of $-0.25 \pm 0.16$ along the extension, and the spectral index remains consistent with free-free emission despite being marginally steeper than optically thin free-free emission. This source did not have previously published $\mathrm{CO}$ or $\mathrm{H}_{2}$ observations, which would indicate outflow direction and the Spitzer data are not resolved well enough to be definitive. However, recent ALMA observations resolved a disk around Per-emb- 8 (J. Tobin et al. 2018, in preparation) and observed the outflow in ${ }^{12} \mathrm{CO}$. The observed disk has a position angle of $45^{\circ}$ and the
CO outflow is orthogonal to this. The extended 4.1 and $6.4 \mathrm{~cm}$ emission has a position angle of about $\sim 45^{\circ}$ different from the CO outflow direction. Thus, like L1448 IRS 3A, the extended radio emission might trace the edge of an ionized outflow cavity or the ionized surface of the disk.

\subsection{Per-emb-18}

Per-emb-18 (Figure 7) is a Class 0 system in the NGC 1333 IRAS7 region consisting of two protostars separated by only $20 \mathrm{au}$ (Tobin et al. 2016b). Thus, C-band observations were unable to resolve the system. The radio emission is asymmetric and the spectral index decreases steeply from $0.37 \pm 0.14$ at the protostellar position to $-1.13 \pm 0.42$ in the southern outflow. Measured position angle of $164^{\circ} \pm 16^{\circ}$ is consistent with the value of the $\mathrm{H}_{2}$ outflow $\left(159^{\circ}\right)$ observed by Davis et al. (2008). The steep negative spectral index in this source is also partly due to the lack of emission detected at $4.1 \mathrm{~cm}$ where extended $6.4 \mathrm{~cm}$ emission is present.

\subsection{Per-emb-20}

Per-emb-20 (Figure 8) is a Class 0 protostar known also as L1455 IRS4. An extension of the 4.1 and $6.4 \mathrm{~cm}$ is observed with a position angle of $137^{\circ} \pm 8^{\circ}$. This position angle is reasonably consistent with the $115^{\circ}$ position angle found by Davis et al. (2008) from $\mathrm{H}_{2}$ observations. The extension is small, but is present at both wavelengths, and other sources in 

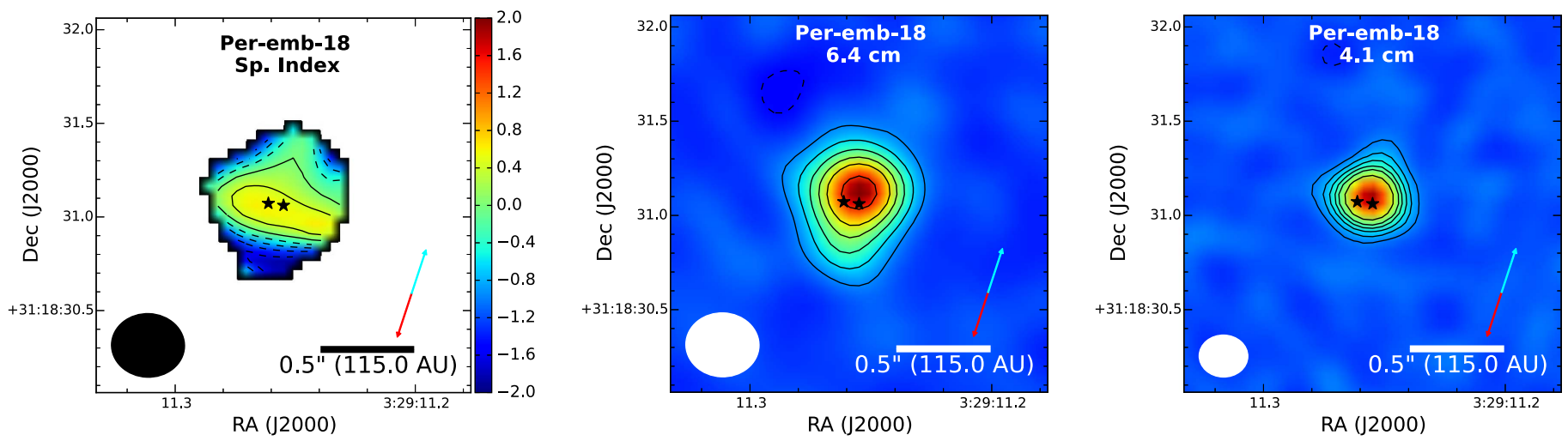

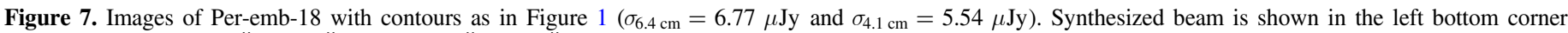

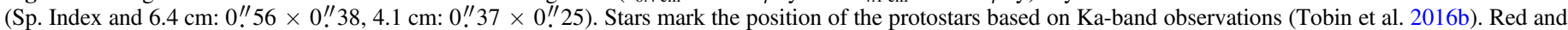
blue arrows indicate outflow direction from Davis et al. (2008) with a position angle of $159^{\circ}$.
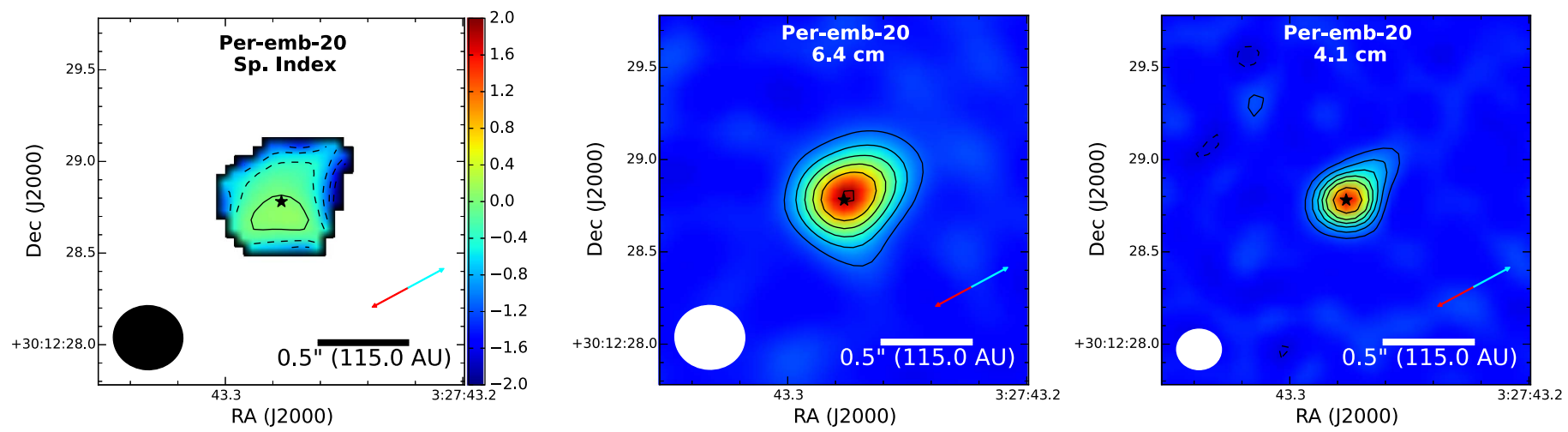

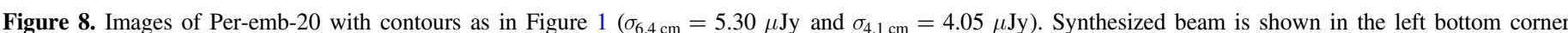

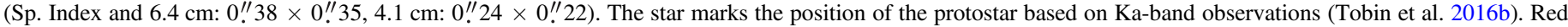
and blue arrows indicate outflow direction from Davis et al. (2008) with a position angle of $115^{\circ}$.

the map do not show a similar extension. The spectral index is flat in the central part $(0.00 \pm 0.18)$ and is decreasing toward the northwest along the extension. The spectral index measured along the extension is $-1.38 \pm 0.51$, but the extension is only marginally resolved at $6.4 \mathrm{~cm}$.

\section{Discussion}

Resolved jets at centimeter wavelengths have typically been associated with massive protostars (e.g., HH 80-81; see Marti et al. 1995) and with a few remarkable low-mass protostars that have exceptionally powerful jets (e.g., L1527, HH 1-2 VLA 1, SVS 13C; see Rodríguez et al. 1997; Rodríguez \& DelgadoArellano 2000; Reipurth et al. 2004). However, analysis of the frequency of extended radio jets toward an unbiased sample of low-mass protostars has only recently become possible with the advent of the upgraded Jansky VLA. While some of the previous studies looked at a reasonably large number of sources (e.g., Anglada et al. 1998), the observations were often conducted at resolutions of $\sim 1^{\prime \prime}$ or greater and the observations were not very sensitive due to the bandwidth limitations of the old VLA. Thus, while some of the protostars observed may have had extended radio jets, the surface brightness sensitivities of the previous observations may have been too low even if the resolution was high enough. Thus, our sensitive, unbiased survey has enabled the detection of eight extended radio jets toward low-mass stars in Perseus, where only one extended radio jet had been previously found (Rodríguez et al. 1997).
The observations presented here provide a more complete picture of extended radio jets in low-mass protostellar systems.

In the study of radio emission toward selected regions with prominent Herbig-Haro objects, Reipurth et al. (2004) showed that almost half of the detected sources have radio jets. Only $11 \%$ of protostars detected in our unbiased survey have resolved emission. It is worth noting that sources of some wellknown HH objects in Perseus (e.g., HH 7-11) appear compact in our observations (Ł. Tychoniec et al. 2018, in preparation).

\subsection{Why Are Some Jets Extended?}

With a larger sample of extended jets, we are able to investigate if there are any evolutionary or morphological trends behind the detection of extended jets. The majority of extended jets are found toward Class 0 protostars, Per-emb-8, Per-emb-18, Per-emb-20, Per-emb-33, SVS 13C, and Per-emb30 which is a borderline Class 0/I source; the others are Class I protostars (Per-emb-36, L1448 IRS 3A). Taking into account a similar number of Class 0 (40) and Class I (38) protostars in the sample, and additional six borderline sources, at first glance it appears that youth tends to play a role in the presence of extended jet emission. Furthermore, the two Class I protostars with extended jets are found within dense regions with Class 0 protostars and dense molecular gas in close proximity. Peremb-20 is the most isolated protostar found with an extended jet. It should be noted that protostellar ages, based on bolometric temperature, are subject to large uncertainties due 
to inclination and reddening. The sources have bolometric luminosities that range between 1 to $10 L_{\odot}$, whereas the most luminous sources in the VANDAM sample (e.g., 4 sources with $L_{\text {bol }}>10 L_{\odot}$ ) do not have extended jets. Thus, extended jets are not directly linked with protostellar luminosity. Geometric orientation of the sources could play a role; for instance, the pole-on alignment would make extended jet emission difficult to observe, but this would prevent the detection of the extended emission of only about $10 \%-20 \%$ of sources based on the probabilities of random orientations. Edge-on orientation, on the other hand, can make sources appear underluminous (Whitney et al. 2003).

It is also possible that extended radio emission could be a transient phenomenon. Strong variability of jets from protostars is supported both with numerical modeling (Machida 2014) and observations (Rodríguez \& Delgado-Arellano 2000). Much less is known about the origin of the variability. Regarding the close relation between accretion and ejection, resolved jets could be induced by recent accretion burst. However, the association of enhanced free-free emission with an accretion burst has not been firmly established given the recent study of Galván-Madrid et al (2015) toward the outbursting source HOPS-383 where an increase in the centimeter flux density was not observed postoutburst. It is possible that the enhanced free-free emission is visible later post-outburst when additional outflow material propagates to greater distances from the protostar.

\subsection{Why Are Some Jets Not Extended?}

While the detection of these extended radio jets is remarkable, it is also remarkable that many sources driving powerful outflows do not have extended radio jets. Famous outflow driving sources such as HH 211 (e.g., Gueth \& Guilloteau 1999), L1448-C (e.g., Bachiller et al. 1990), SVS 13A, NGC 1333 IRAS 2A, NGC 1333 IRAS 4A/B (e.g., Plunkett et al. 2013) only show compact freefree emission. These sources are at a variety of inclination angles, so the pole-on viewing should not be a systematic problem. They are all also very young sources and are located in regions with high protostellar density, high surrounding gas density, and/or nearby sources driving extended jets. Furthermore, some of these sources have high-velocity molecular emission features located near the protostar position, indicating that energies are high enough for shocks to produce extended free-free emission on these scales. Thus, it remains unclear why some of these powerful outflow sources do not have extended jets, while some less energetic sources do. Our observations are sensitive to structures as large as $\sim 9^{\prime \prime}$, thus any structure in the crucial region close to the protostar should be detectable up to the sensitivity limit.

It is possible that those sources have high-density material surrounding them, as indicated by vast amount of entrained gas in their molecular outflows. Higher densities could result in effective molecular cooling and rapid recombination that will keep the level of ionization low, hampering the detection of the free-free emission. Rapid cooling in the protostellar envelope also implicates that for extended jets, heating occurs in situ by internal shocks, thus it is likely that more variable jets, by producing more internal shocks, are the sources of extended radio emission. It would explain the lack of relation between the molecular outflow strength and the presence of extended radio jet, as the former is expected to be less sensitive to short-time variations.

Recent observations with Herschel provided new insights into the properties of jets. Nisini et al. (2015) used observations of [O I] to probe the atomic component of outflows. Such observations allowed to describe a process of the jet evolution. In this scheme, the molecular jet would be progressively dissociated, becoming mostly atomic, and eventually appear as hot and ionized. Radio observations present a unique tool to study the base of the protostellar jet during the most embedded phase. It appears that the ionized component of the outflow can be present at very early stages of star formation. Lahuis et al. (2010) observed signatures of the ionization with Spitzer, but without the ability to disentangle the processes spatially. From our observations, we can show that ionization can frequently happen away from the protostar, although we are not able to say whether this happens in the cavity walls or in the spot shocks.

\subsection{Resolved Emission Not Aligned With the Outflow}

Per-emb-8 and L1448 IRS 3A uniquely have misaligned radio emission relative to their molecular outflows. While jets are known to precess (e.g., L1157; Gueth et al. 1996; Looney et al. 2007), precession angles are typically small for low-mass protostars (Frank et al. 2014) while a massive protostar has shown changes in the jet direction of up to $\sim 45^{\circ}$ (Cunningham et al. 2009). Thus, it is possible, but perhaps not likely, that jet precession is the cause of the misalignment between the outflow and radio emission for these two sources. We note that a water maser had been previously identified toward the L1448 IRS3 region with the $37 \mathrm{~m}$ Haystack Telescope (Anglada et al. 1989), but the maser faded before followup with the VLA, so exact positional relation with the extended radio jet cannot be determined.

An additional possibility is that we are tracing an ionized portion of an outflow cavity or disk surface, where there is a lowextinction pathway from the protostar emitting UV radiation to the cavity or disk surface. Both Per-emb-8 and L1448 IRS 3A have well-developed disks observed in the dust continuum and molecular lines (J. Tobin et al. 2018, in preparation). Moreover, toward more massive protostar Serpens SMM1, Hull et al. (2016) recently discovered extended radio emission from the outflow cavity walls. While SMM1 is a much more luminous source $\left(\sim 100 L_{\odot}\right.$; Kristensen et al. 2012) and the radio emission is extended over a much larger area, we suggest that we are observing a similar phenomenon on much smaller scales toward these two low-mass protostars.

\subsection{Significance of Synchrotron Emission}

Toward five of eight protostars in our sample of extended radio jets, we detect negative spectral index in the positions away from the protostar. To date, this phenomenon has only been observed within outflows of massive protostars (e.g., Serpens SMM1, HH 80-81; see Curiel et al. 1993 and Marti et al. 1995), one solar-mass Class II protostar (DG Tau; Ainsworth et al. 2014), and a single example of Class I lowmass protostar (L778 VLA5; Girart et al. 2002). In our observation, we see the tentative detection of synchrotron emission in the outflow of low-mass Class 0/I protostars. Of the entire sample, the most robust detection of synchrotron emission offset from the protostar position is found toward Peremb-36. It has visibly shifted peak emission at $6.4 \mathrm{~cm}$, presumably associated with the intensely shocked material. This shock may be strong enough to produce cosmic rays.

In the study of Serpens SMM1, an intermediate-mass Class 0 protostar powering radio jet with negative spectral indices, (Rodríguez-Kamenetzky et al. 2016) showed that conditions in this jet allows the production of the cosmic rays by diffuse 
shock acceleration (DSA; e.g., Drury 1991). They suggested that this process might be episodic and, for example, a close binary encounter might trigger jet velocity enhancement, creating favorable conditions for the cosmic rays production.

The presence of synchrotron emission being generated within the star-forming core could have significant implications on the chemistry of the gas associated with disk and planet formation. Recent work has shown that high-energy cosmic rays may be attenuated by the magnetic field of the young stars (Cleeves et al. 2013), leaving only radioactive decay as a source of ionization inside protoplanetary disks. While that model was specific to more evolved pre-main sequence stars with disks, Padovani et al. $(2013,2014)$ show that magnetic fields in collapsing protostellar clouds can also shield cosmic rays from penetrating too deeply into clouds.

Thus, the potential lack of ionization in these clouds will strongly affect the chemical composition of the gas in the diskforming region of the clouds. However, Padovani et al. (2016) demonstrated that cosmic rays can be accelerated to relativistic energies through first-order Fermi acceleration (DSA) even at jet velocities as low as $100 \mathrm{~km} \mathrm{~s}^{-1}$, typical for low-mass outflows. Thus, if there is a component of synchrotron emission in some of these extended jets, it could be acting as a local source of cosmic rays, enabling richer chemistry (e.g., Aikawa et al. 1997; Padovani et al. 2013; Eistrup et al. 2016; RodgersLee et al. 2017).

It should be noted that for some of the sources, the uncertainty of the calculated spectral index value is high $(\sim 0.5)$, so we cannot completely rule out the possibility that the emission is optically thin free-free. Rodríguez et al. (1993) showed that indices below -0.1 are virtually impossible for thermal processes, which suggests that at least some of our sources are strong candidates for synchrotron emission.

In the positions of the shocks, we may also find associated X-ray emission (Pravdo et al. 2004), such as was found toward DG Tau (Güdel et al. 2008). The only protostar within our sample with sensitive X-ray data is SVS 13C, and it was undetected. Per-emb-36 lies at the outskirts of the X-ray map taken toward NGC 1333, but the X-ray emission toward its position cannot be positively associated with it due to the nearby, more evolved star BD +30 547. Non-detections have also been reported toward both Per-emb-33 and L1448 IRS 3A by Tsujimoto et al. (2005).

\section{Conclusions}

We conducted observations of all known Class 0 and I protostars in Perseus molecular cloud in 4.1 and $6.4 \mathrm{~cm} .8$ of 71 detected sources exhibit resolved radio emission. In this paper, we analyzed emission toward the resolved subset of sources with the following conclusions:

1. We observe resolved emission toward $11 \%$ sources from the unbiased radio survey. Their integrated spectral indices are consistent with optically thin free-free emission; however, we find them systematically lower than the median for the whole sample.

2. Toward five sources-Per-emb-18, Per-emb-20, Per-emb-30, Per-emb-36, and L1448 IRS 3A-we detect negative spectral indices in the outflow position indicative of synchrotron emission. Per-emb-36 is the most robust candidate for synchrotron emission and its peak of emission is shifted away from the protostar, indicative of a strong shock. This is one of the few detections of synchrotron emission toward a lowmass protostar. This result suggests that production of cosmic rays might be frequent, however transient, in the shocks of low-mass protostars, which could have significant repercussions for disk and planet formation.

3. Six sources have position angles matching directions of the large-scale molecular outflow, showing that in most cases radio emission can be interpreted as an ionized base of the jet.

4. Two sources, Per-emb-8 and L1448 IRS 3A, show significant misalignment of the resolved radio emission. We suggest that this emission not necessarily comes from the jet, but more likely from the ionized outflow cavity wall or even the upper layer of the disk.

Since the upgrade of the VLA, we are able to study ionized component of the protostellar outflow with high resolution for an unprecedented number of protostars. This study showed that analysis of the spectral index and alignment of the thermal radio jet can be a useful tool to examine the most embedded protostars. With the James Webb Space Telescope, we will be able to resolve emission from ions and atoms in the direct vicinity of the protostar, which will allow us to disentangle different processes. Future $\mathrm{cm}$-wave facilities will provide even higher resolution and sensitivity to show if negative spectral indices toward jets of low-mass protostars are common phenomena.

The authors thank the anonymous referee for comments that improved the clarity of the paper. Ł.T. is supported by Leiden/ ESA Astrophysics Program for Summer Students (LEAPS). Astrochemistry in Leiden is supported by the Netherlands Research School for Astronomy (NOVA), by a Royal Netherlands Academy of Arts and Sciences (KNAW) professor prize, and by the European Union A-ERC grant 291141 CHEMPLAN. A.K. acknowledges support from the Foundation for Polish Science (FNP) and the Polish National Science Center grants 2013/11/N/ST9/00400 and 2016/21/D/ST9/01098. Ł.T. and A.K. acknowledge support from the HECOLS International Associated Laboratory, supported in part by the Polish NCN grant DEC-2013/08/M/ST9/00664. ZYL is supported in part by NASA NNX 14AB38G, and NSF AST1313083, AST-1716259. The National Radio Astronomy Observatory is a facility of the National Science Foundation operated under cooperative agreement by Associated Universities, Inc. This research made use of: Astropy, a communitydeveloped core Python package for Astronomy (Astropy Collaboration 2013, http://astropy.org); APLpy, an opensource plotting package for Python hosted at http://aplpy. github.com; Matplotlib library (Hunter 2007); and NASA's Astrophysics Data System.

\section{Appendix Additional Plots}

Here Figures 9-16 show the positions where the spectral index was measured, the corresponding error map of the spectral index, and the full-bandwidth image centered at $5 \mathrm{~cm}$. 

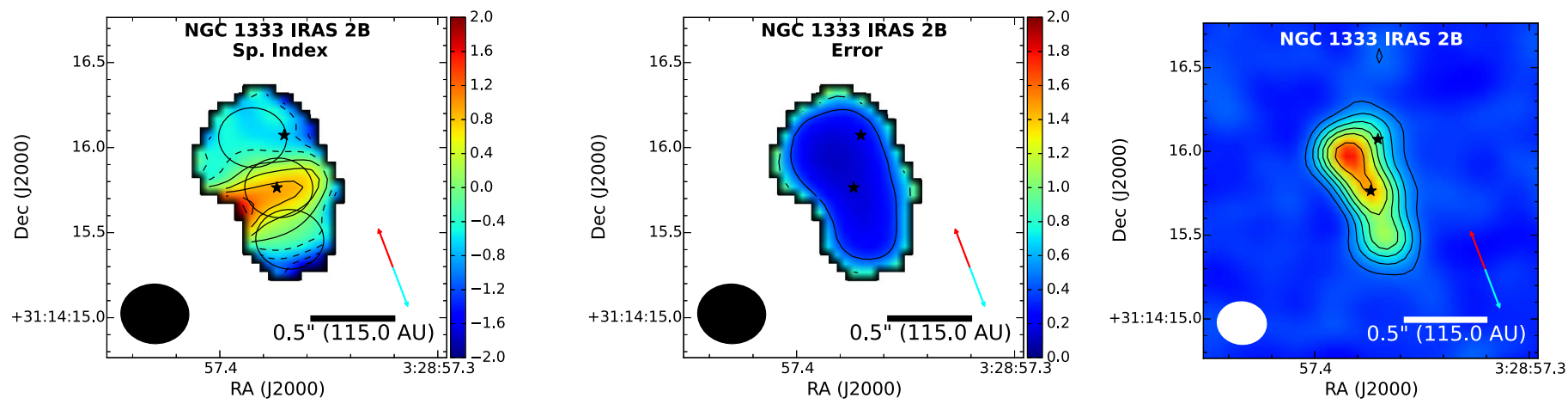

Figure 9. Maps of IRAS 2B. Left: spectral index map with marked positions where median flux was measured to calculate spectral indices. Center: spectral index error map with contours $[0.4,0.8,1.2,1.6,2.0]$. Right: full-bandwidth image centered at $6.05 \mathrm{GHz}(5 \mathrm{~cm})$. Contours as in Figure $1\left(\sigma_{5} \mathrm{~cm}=3.77 \mu \mathrm{Jy}\right)$. Synthesized beam is shown in the left bottom corner $\left(0 !^{\prime \prime} 29^{\prime \prime} \times 0 !^{\prime \prime} 25\right)$. Stars mark the positions of the protostars based on Ka-band observations (Tobin et al. 2016b). Red and blue arrows indicate outflow direction from Plunkett et al. (2013).
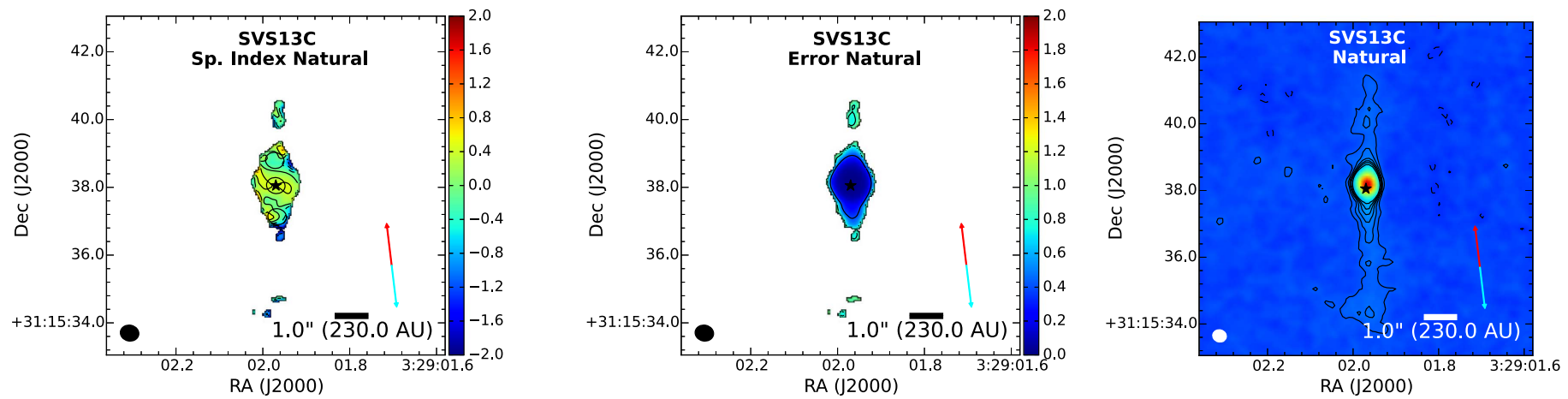

Figure 10. Same as Figure 9, but for SVS $13 \mathrm{C}\left(\sigma_{5} \mathrm{~cm}=3.06 \mu \mathrm{Jy}\right)$. Synthesized beam of the full-bandwidth image is 0 .! $41 \times 0$." 36 . The star marks the position of the protostar based on Ka-band observations (Tobin et al. 2016b). Red and blue arrows indicate outflow direction from Plunkett et al. (2013).
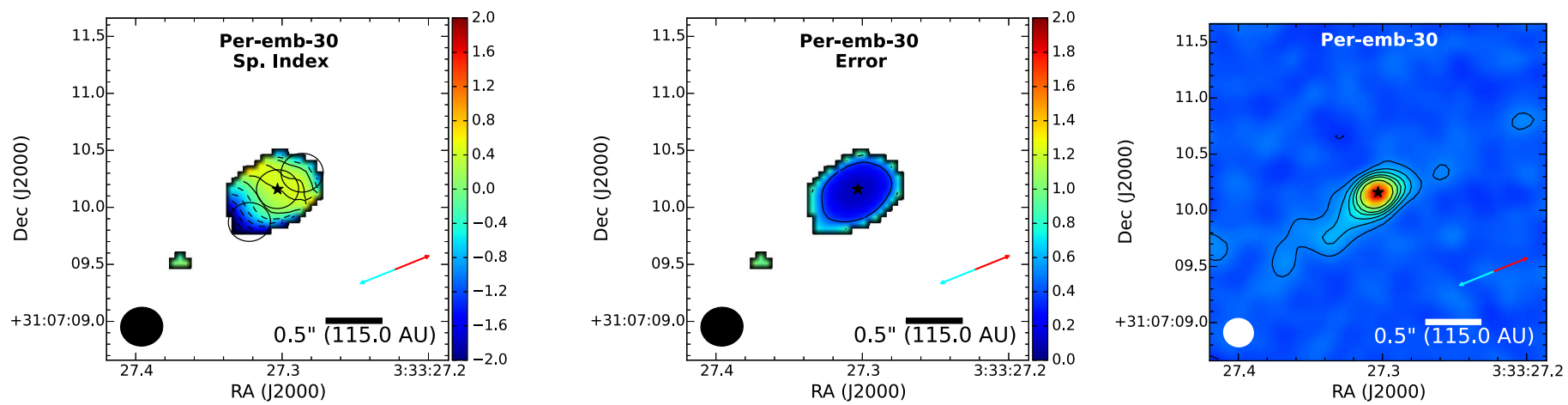

Figure 11. Same as in Figure 9, but for Per-emb-30 $\left(\sigma_{5 \mathrm{~cm}}=3.58 \mu \mathrm{Jy}\right)$. Synthesized beam of the full-bandwidth image is $0.27 \times 0$." 25$)$. The star marks the position of the protostar based on Ka-band observations (Tobin et al. 2016b). Red and blue arrows indicate the outflow direction from Davis et al. (2008).
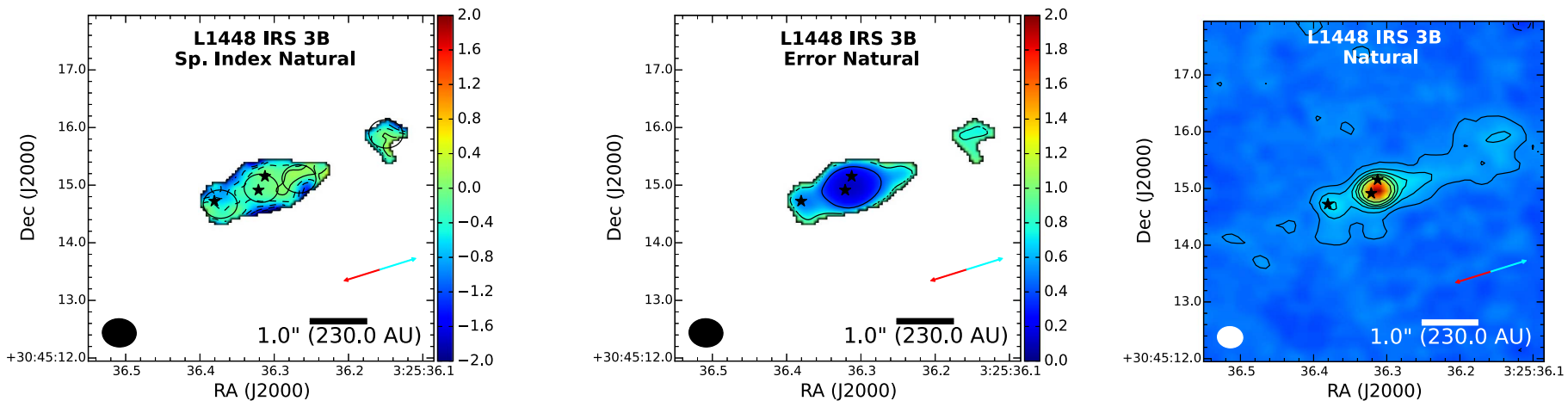

Figure 12. Same as Figure 9, but for L1448 IRS 3B $\left(\sigma_{5 \mathrm{~cm}}=3.35 \mu \mathrm{Jy}\right)$. The synthesized beam of the full-bandwidth image is $\left.0 . .45 \times 0 . " 37\right)$. Stars marks the position of the protostar based on Ka-band observations (Tobin et al. 2016b). Red and blue arrows indicate outflow direction from Lee et al. (2015). 

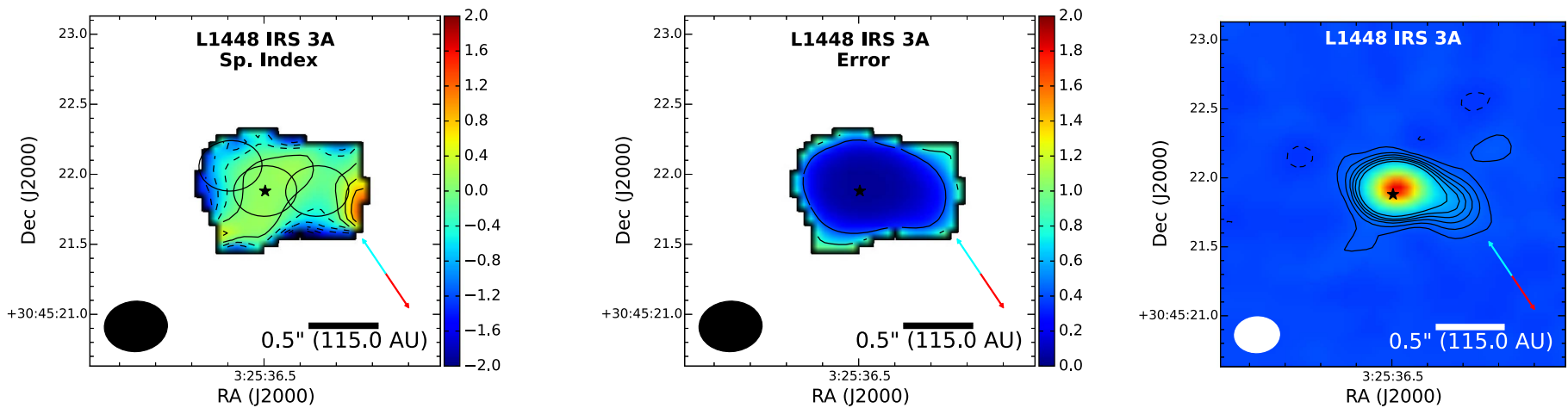

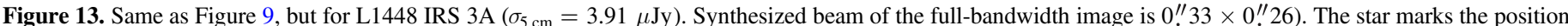
of the protostar based on Ka-band observations. Red and blue arrows indicate outflow direction from Lee et al. (2015).
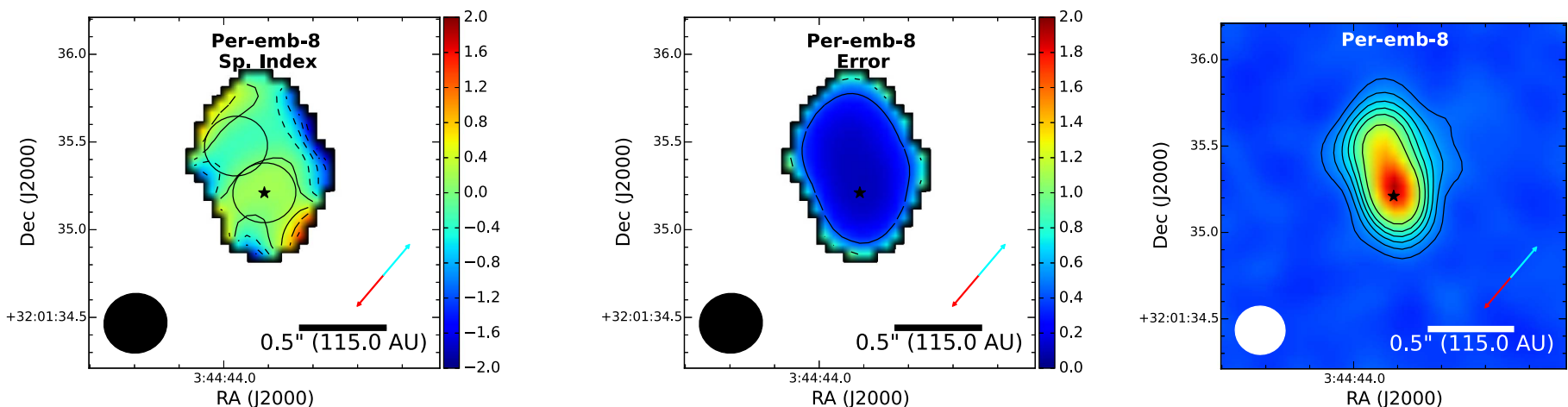

Figure 14. Same as Figure 9, but for Per-emb-8. Contours as in Figure $1\left(\sigma_{5 \mathrm{~cm}}=3.34 \mu \mathrm{Jy}\right)$. The synthesized beam of the full-bandwidth image is 0 !' $29 \times 0$.! 28$)$. The star marks the position of the protostar based on Ka-band observations (Tobin et al. 2016b). Red and blue arrows indicate outflow direction of $135^{\circ}$ based on ALMA observations (outflow is assumed to be perpendicular to the disk (J. Tobin 2018, in preparation).
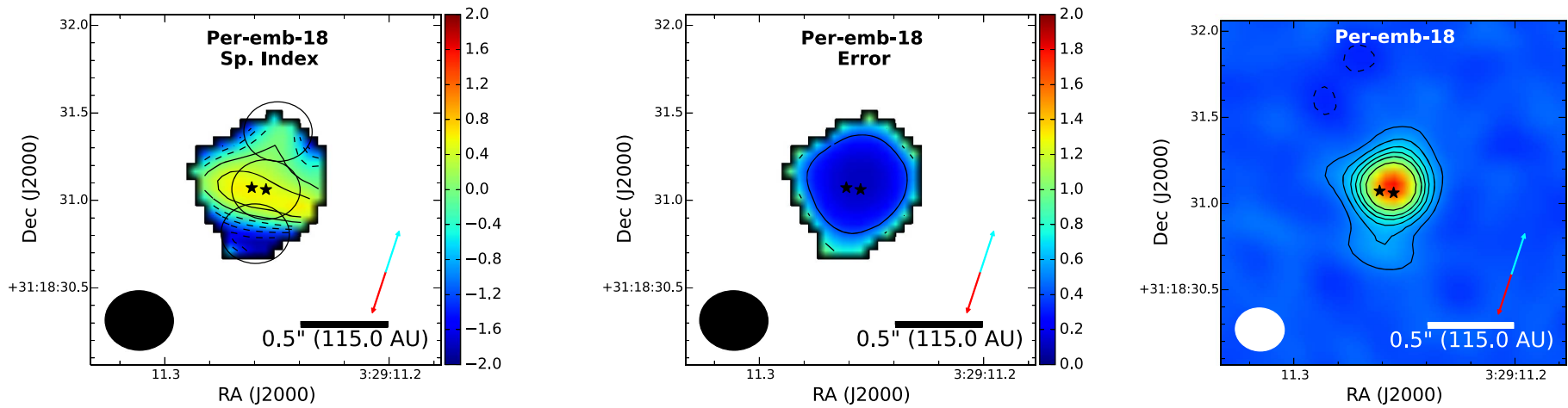

Figure 15. Same as Figure 9, but for Per-emb-18. Contours as in Figure $1\left(\sigma_{5} \mathrm{~cm}=3.34 \mu \mathrm{Jy}\right)$. The synthesized beam of the full-bandwidth image is $\left.0 " 29 \times 0 / 28\right)$. Stars mark the positions of the protostar based on Ka-band observations (Tobin et al. 2016b). Red and blue arrows indicate outflow direction from Davis et al. (2008).
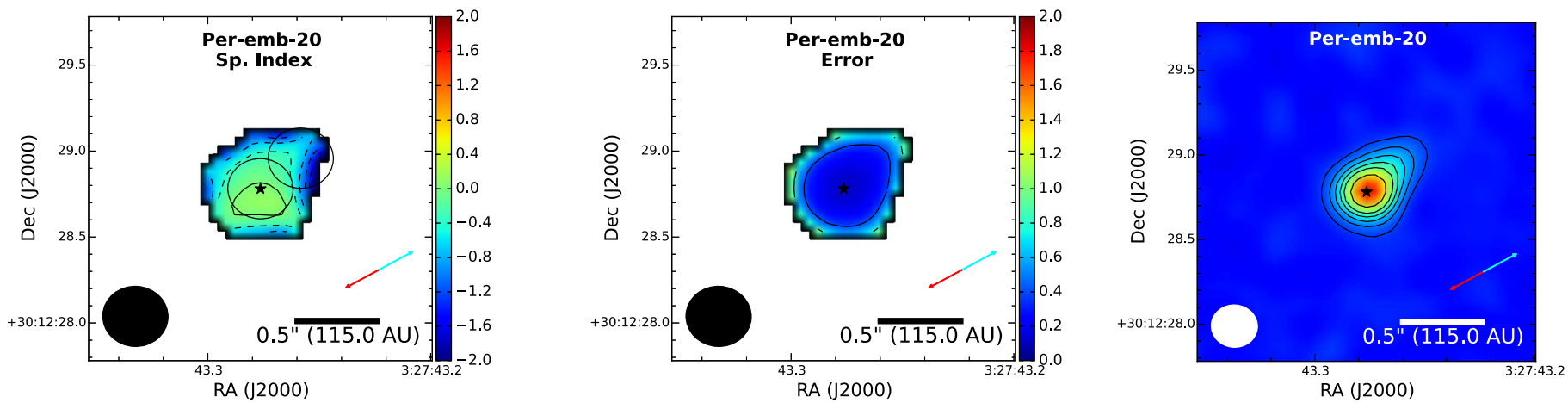

Figure 16. Same as Figure 9, but for Per-emb-20. Contours as in Figure $1\left(\sigma_{5} \mathrm{~cm}=3.68 \mu \mathrm{Jy}\right)$. Synthesized beam of the full-bandwidth image is 0 .' $27 \times 0$.' 25$)$. The star marks the position of the protostar based on Ka-band observations (Tobin et al. 2016b). Red and blue arrows indicate outflow direction from Davis et al. (2008). 


\section{ORCID iDs}

Łukasz Tychoniec (1) https://orcid.org/0000-0002-9470-2358

John J. Tobin (1) https://orcid.org/0000-0002-6195-0152

Agata Karska (ํ) https://orcid.org/0000-0001-8913-925X

Claire Chandler (ib https://orcid.org/0000-0002-7570-5596

Michael M. Dunham (i) https://orcid.org/0000-0003-

0749-9505

Leslie W. Looney (1) https://orcid.org/0000-0002-4540-6587

Carl Melis 이 https://orcid.org/0000-0001-9834-7579

Sarah I. Sadavoy (1) https://orcid.org/0000-0001-7474-6874

\section{References}

Aikawa, Y., Umebayashi, T., Nakano, T., \& Miyama, S. M. 1997, ApJL, 486, L51

Ainsworth, R. E., Scaife, A. M. M., Ray, T. P., et al. 2014, ApJL, 792, L18

AMI Consortium: Scaife, A. M. M., Buckle, J. V., Ainsworth, R. E., et al. 2012a, MNRAS, 420, 3334

AMI Consortium: Scaife, A. M. M., Curtis, E. I., Davies, M., et al. 2011a, MNRAS, 410, 2662

AMI Consortium: Scaife, A. M. M., Hatchell, J., Davies, M., et al. 2011b, MNRAS, 415, 893

AMI Consortium: Scaife, A. M. M., Hatchell, J., Davies, M., et al. 2012b, MNRAS, 420, 1019

Anglada, G. 1995, RMxAA, 1, 67

Anglada, G., Rodriguez, L. F., Torrelles, J. M., et al. 1989, ApJ, 341, 208

Anglada, G., Villuendas, E., Estalella, R., et al. 1998, AJ, 116, 2953

Astropy Collaboration, Robitaille, T. P., Tollerud, E. J., et al. 2013, A\&A, 558,33

Bachiller, R., Martin-Pintado, J., Tafalla, M., Cernicharo, J., \& Lazareff, B. 1990, A\&A, 231, 174

Bally, J., Devine, D., \& Reipurth, B. 1996, ApJL, 473, L49

Bontemps, S., Andre, P., Terebey, S., \& Cabrit, S. 1996, A\&A, 311, 858

Cabrit, S., \& Bertout, C. 1992, A\&A, 261, 274

Caratti o Garatti, A., Garcia Lopez, R., Antoniucci, S., et al. 2012, A\&A, 538, A64

Carrasco-González, C., Anglada, G., Rodríguez, L. F., Torrelles, J. M., \& Osorio, M. 2008, AJ, 136, 2238

Carrasco-González, C., Rodríguez, L. F., Anglada, G., et al. 2010, Sci, 330, 1209

Chen, H., Myers, P. C., Ladd, E. F., \& Wood, D. O. S. 1995, ApJ, 445, 377

Chiang, H.-F., Looney, L. W., \& Tobin, J. J. 2012, ApJ, 756, 168

Cleeves, L. I., Adams, F. C., \& Bergin, E. A. 2013, ApJ, 772, 5

Condon, J. J., Cotton, W. D., Greisen, E. W., et al. 1998, AJ, 115, 1693

Cunningham, N. J., Moeckel, N., \& Bally, J. 2009, ApJ, 692, 943

Curiel, S., Raymond, J. C., Moran, J. M., Rodriguez, L. F., \& Canto, J. 1990, ApJL, 365, L85

Curiel, S., Rodriguez, L. F., Moran, J. M., \& Canto, J. 1993, ApJ, 415, 191

Davis, C. J., Scholz, P., Lucas, P., Smith, M. D., \& Adamson, A. 2008, MNRAS, 387, 954

Drury, L. O. 1991, MNRAS, 251, 340

Dzib, S. A., Loinard, L., Mioduszewski, A. J., et al. 2013, ApJ, 775, 63

Dzib, S. A., Loinard, L., Rodríguez, L. F., et al. 2015, ApJ, 801, 91

Eistrup, C., Walsh, C., \& Van Dishoeck, E. F. 2016, A\&A, 595, A83

Enoch, M. L., Evans, N. J., II, Sargent, A. I., \& Glenn, J. 2009, ApJ, 692, 973

Frank, A., Ray, T. P., Cabrit, S., et al. 2014, in Protostars and Planets VI, ed. H. Beuther (Tucson, AZ: Univ. Arizona Press), 451

Galván-Madrid, R., Rodríguez, L. F., Liu, H. B., et al. 2015, ApJL, 806, L32

Girart, J. M., Curiel, S., Rodríguez, L. F., \& Cantó, J. 2002, RMxAA, 38, 169

Güdel, M., Skinner, S. L., Audard, M., Briggs, K. R., \& Cabrit, S. 2008, A\&A, 478, 797

Gueth, F., \& Guilloteau, S. 1999, A\&A, 343, 571

Gueth, F., Guilloteau, S., \& Bachiller, R. 1996, A\&A, 307, 891

Hirota, T., Bushimata, T., Choi, Y. K., et al. 2008, PASJ, 60, 37

Hull, C. L. H., Girart, J. M., Kristensen, L. E., et al. 2016, ApJL, 823, L27
Hunter, J. D. 2007, CSE, 9, 90

Jørgensen, J. K., Johnstone, D., Kirk, H., \& Myers, P. C. 2007, ApJ, 656, 293

Karska, A., Kristensen, L. E., van Dishoeck, E. F., et al. 2014, A\&A, 572, A9

Kounkel, M., Hartmann, L., Loinard, L., et al. 2014, ApJ, 790, 49

Kristensen, L. E., van Dishoeck, E. F., Bergin, E. A., et al. 2012, A\&A, 542, A8

Kwon, W., Looney, L. W., Crutcher, R. M., \& Kirk, J. M. 2006, ApJ, 653, 1358

Lahuis, F., van Dishoeck, E. F., Jørgensen, J. K., Blake, G. A., \& Evans, N. J. 2010, A\&A, 519, A3

Lee, K. I., Dunham, M. M., Myers, P. C., et al. 2015, ApJ, 814, 114

Lee, K. I., Dunham, M. M., Myers, P. C., et al. 2016, ApJL, 820, L2

Lee, K. I., Fernández-López, M., Storm, S., et al. 2014, ApJ, 797, 76

Looney, L. W., Mundy, L. G., \& Welch, W. J. 2000, ApJ, 529, 477

Looney, L. W., Tobin, J. J., \& Kwon, W. 2007, ApJL, 670, L131

Machida, M. N. 2014, ApJL, 796, L17

Marti, J., Rodriguez, L. F., \& Reipurth, B. 1993, ApJ, 416, 208

Marti, J., Rodriguez, L. F., \& Reipurth, B. 1995, ApJ, 449, 184

McMullin, J. P., Waters, B., Schiebel, D., Young, W., \& Golap, K. 2007, in ASP Conf. Ser. 376, Astronomical Data Analysis Software and Systems XVI, ed. R. A. Shaw, F. Hill, \& D. J. Bell (San Francisco, CA: ASP), 127

Nisini, B., Giannini, T., \& Lorenzetti, D. 2002, ApJ, 574, 246

Nisini, B., Santangelo, G., Giannini, T., et al. 2015, ApJ, 801, 121

Ortiz-León, G. N., Loinard, L., Mioduszewski, A. J., et al. 2015, ApJ, 805, 9

Padovani, M., Galli, D., Hennebelle, P., Commerçon, B., \& Joos, M. 2014, A\&A, 571, A33

Padovani, M., Hennebelle, P., \& Galli, D. 2013, A\&A, 560, A114

Padovani, M., Marcowith, A., Hennebelle, P., \& Ferrière, K. 2016, A\&A, 590, A8

Panagia, N., \& Felli, M. 1975, A\&A, 39, 1

Pech, G., Loinard, L., Dzib, S. A., et al. 2016, ApJ, 818, 116

Perley, R. A., \& Butler, B. J. 2017, ApJS, 230, 7

Plunkett, A. L., Arce, H. G., Corder, S. A., et al. 2013, ApJ, 774, 22

Pravdo, S. H., Tsuboi, Y., \& Maeda, Y. 2004, ApJ, 605, 259

Preibisch, T. 1997, A\&A, 324, 690

Raga, A. C., Noriega-Crespo, A., Carey, S. J., \& Arce, H. G. 2013, AJ, 145, 28

Reipurth, B., \& Cernicharo, J. 1995, RMxAA, 1, 43

Reipurth, B., Rodríguez, L. F., Anglada, G., \& Bally, J. 2002, ApJ, 124, 1045

Reipurth, B., Rodríguez, L. F., Anglada, G., \& Bally, J. 2004, AJ, 127, 1736

Reynolds, S. P. 1986, ApJ, 304, 713

Rodgers-Lee, D., Taylor, A. M., Ray, T. P., \& Downes, T. P. 2017, MNRAS, 472, 26

Rodríguez, L. F. 1994, RMxAA, 29, 69

Rodríguez, L. F., Anglada, G., \& Curiel, S. 1997, ApJL, 480, L125

Rodríguez, L. F., Anglada, G., \& Curiel, S. 1999, ApJS, 125, 427

Rodríguez, L. F., Canto, J., Torrelles, J. M., \& Ho, P. T. P. 1986, ApJL, 301, L25

Rodríguez, L. F., Curiel, S., Moran, J. M., et al. 1989, ApJL, 346, L85

Rodríguez, L. F., Delgado-Arellano, V. G., Gómez, Y., et al. 2000, AJ, 119,882

Rodríguez, L. F., Marti, J., Canto, J., Moran, J. M., \& Curiel, S. 1993, RMxAA, 25, 23

Rodríguez-Kamenetzky, A., Carrasco-González, C., Araudo, A., et al. 2016, ApJ, 818, 27

Sadavoy, S. I., Di Francesco, J., André, P., et al. 2014, ApJL, 787, L18

Shirley, Y. L., Claussen, M. J., Bourke, T. L., Young, C. H., \& Blake, G. A. 2007, ApJ, 667, 329

Snell, R. L., \& Bally, J. 1986, ApJ, 303, 683

Storm, S., Mundy, L. G., Fernández-López, M., et al. 2014, ApJ, 794, 165

Tobin, J. J., Dunham, M. M., Looney, L. W., et al. 2015, ApJ, 798, 61

Tobin, J. J., Kratter, K. M., Persson, M. V., et al. 2016a, Natur, 538, 483

Tobin, J. J., Looney, L. W., Li, Z.-Y., et al. 2016b, ApJ, 818, 73

Tsujimoto, M., Kobayashi, N., \& Tsuboi, Y. 2005, AJ, 130, 2212

Whitney, B. A., Wood, K., Bjorkman, J., \& Wolff, M. J. 2003, ApJ, 591, 1049

Wilner, D. J., D’Alessio, P., Calvet, N., Claussen, M. J., \& Hartmann, L. 2005, ApJL, 626, L109

Wu, Y., Wei, Y., Zhao, M., et al. 2004, A\&A, 426, 503 